\title{
Nutrition facts and limits for micronutrients in tree species used in urban forestry
}

\author{
FLÁVIA G.K. BRUN ${ }^{1}$, ELEANDRO J. BRUN ${ }^{1}$, DIONATAN GERBER ${ }^{1}$, DENISE A. SZYMCZAK ${ }^{2}$, \\ EDUARDO K. LONDERO ${ }^{3}$, EVANDRO A. MEYER ${ }^{4}$ and MÁRCIO C. NAVROSKI ${ }^{5}$
}

\author{
${ }^{1}$ Programa de Pós-Graduação em Agroecossistemas, Universidade Tecnológica Federal do Paraná, Campus \\ Dois Vizinhos, Estrada para Boa Esperança, Km 4, 85660-000 Dois Vizinhos, PR, Brazil \\ ${ }^{2}$ Universidade Tecnológica Federal do Paraná, Campus Francisco Beltrão, Linha \\ Santa Bárbara, s/n, 85601-970 Francisco Beltrão, PR, Brazil \\ ${ }^{3}$ Companhia TANAC S.A., Departamento de Pesquisa Florestal, Rua Torbjorn Weibull, 199, 95780-000 Montenegro, RS, Brazil \\ ${ }^{4}$ Programa de Pós-Graduação em Engenharia Florestal, Universidade Federal de Santa \\ Maria, Avenida Roraima, 1000, Camobi, 97105-900 Santa Maria, RS, Brazil \\ ${ }^{5}$ Programa de Pós-Graduação em Engenharia Florestal, Universidade do Estado de Santa Catarina, Centro de \\ Ciências Agroveterinárias, Av. Luiz de Camões, 2090, Conta Dinheiro, 88520-000 Lages, SC, Brazil
}

Manuscript received on December 5, 2016; accepted for publication on April 11, 2017

\begin{abstract}
There is a huge lack of researches that evaluate the nutritional limits in tree species used in urban forestry, especially in terms of micronutrients. This study aimed to establish limits and range of micronutrients levels for the proper development of tree species utilized in urban forestry. The study was conducted in the city of Santa Maria-RS-Brazil. Through forest inventory, 23 forest species present in urban forest were selected, and 05 vegetative branches of each tree were collected, in which the contents of $\mathrm{B}, \mathrm{Cu}, \mathrm{Fe}, \mathrm{Mn}$ and $\mathrm{Zn}$ were analyzed. Ranges of micronutrients' contents were developed for class limits criteria. Nutritional problems were detected for $\mathrm{B}, \mathrm{Cu}$ and $\mathrm{Zn}$ in G. robusta and S. cumini, indicating a need of fertilization and management of these trees. The levels of Mn were within an adequate range only for the species $C$. illinoensis and $H$. chrysotrichus. The contents of $\mathrm{B}$ were higher than the level considered adequate for $H$. chrysotrichus and M. nigra. The rates of Fe showed high levels for E. japonica, H. chrysotrichus and $S$. babylonica. The estimated nutritional limits enable a greater control in the classification of the results for each tree species utilized in urban forestry.
\end{abstract}

Key words: forest species, urban forestry, forest nutrition.

\section{INTRODUCTION}

The urban trees play an important role in the wellbeing of urban population, illustrating one of the connecting links between man and nature, contributing in a significant manner as

Correspondence to: Eleandro José Brun

E-mail: eleandrobrun.utfpr@gmail.com a fundamental element for the equilibrium of the artificial environment where it is located. Therefore, it does not only play roles related to the environment's beautification but also has the ability of fulfilling relevant ecologic functions (Kern and Schmitz 2013).

In urban planning, it is necessary to acquire knowledge about the trees species, which can be 
obtained by conducting a forest inventory, tool that gather different and accurate information about variables related to urban trees, including the content and distribution of nutrients on them. This information provides, thus, guidance to the public manager responsible for the fertilization and management of the tree species utilized.

Studies about the determination of nutritional limits in the trees' biomass components may be applied as indicators of possible environmental impacts from trees on nutrients available in the soil and other potential sources in urban centres, as well as the possible impacts from these on the trees. Based on the examination of these relationships, it is feasible to adopt management strategies that conduct to a loss reduction (Caldeira et al. 2006) and to a better utilization of the nutrients present in the urban environment from trees of different species.

When young trees are not or are inadequately fertilized, there is often a need for fertilization to occur in adulthood through techniques such as surface or net fertilization, liquid injection in holes, and other methods (Lilly 2015). Proof of this is the fact that species with nutritional deficiencies were found in this work, carried out with adult trees.

In general, the nutritional behavior of the isolated tree species planted in urban forest is different in relation to the same species in one forests, because the influence of factors related to urbanization interferes in order to make many species differentiated in their ability to absorb, translocate and store nutrients in their tissues. This happens because factors such as air, rainwater and soil pollution that cause many trees absorb nutrients and also other elements in different amounts of their physiological needs, being able to have deficiency as well as toxicity of some elements.

Researches associated with the micronutrients levels in tree species located in the street arborisation in Brazil are scarce, mostly in terms of establishing quantitative patterns directly applicable to urban trees management. Then, the present study aimed to establish limits and range of micronutrients contents for the proper development of tree species utilized in urban forestry in cities located in the South Region of Brazil.

\section{MATERIALS AND METHODS}

The study was realized taking as sample basis the streets in the Camobi neighborhood, in the city of Santa Maria, state of Rio Grande do Sul, Brazil. This neighborhood is localized between the coordinates $29^{\circ} 42^{\prime} 02^{\prime \prime}-29^{\circ} 42^{\prime} 30^{\prime \prime} \mathrm{S}$ and $53^{\circ} 42^{\prime} 20^{\prime \prime}$

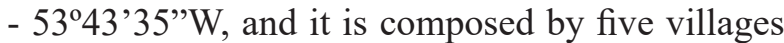
and three avenues, respectively: Santos Dumont, Santa Tereza, Assunção, Estação Colônia, Santa Helena Villages and Prefeito Evandro Behr, Roraima and Prefeito Rodolfo Behr Avenues.

The soil belongs to the mapping unit RedYellow Ultisol (EMBRAPA 2006). The climate of the region is classified as subtropical temperate (Cfa), with hot summer, according to classification of Köppen, characterized by a mean annual temperature between 18.0 and $20.0^{\circ} \mathrm{C}$. The rains are well distributed throughout the year, being that the average annual rainfall is between 1,600 and 1,900 mm (Alvares et al. 2013).

For the study, all of the individuals present in the neighborhood were selected, which resulted in 23 exotic and native species. Those species were evaluated regarding their quali-quantitative characteristics gathered through forest inventory (Szymczak et al. 2012).

The studied species were the following: Campomanesia xanthocarpa O. Berg, Carya illinoensis (Wangenh.) K. Koch, Eriobotrya japonica Lindl., Eugenia involucrata DC., Eugenia uniflora L., Grevillea robusta A. Cunn. ex R. Br., Handroanthus chrysotrichus (Mart. Ex A. DC.), Handroanthus heptaphyllus (Mart.) Mattos, Inga marginata Willd., Jacaranda mimosifolia D. Don, Lagerstroemia indica L., Melia azedarach L., 
Morus nigra L., Peltophorum dubium (Spreng.) Taub., Poincianella pluviosa var. peltophoroides (Benth.) L. P. Queiroz., Psidium cattleyanum Sabine, Psidium guajava L., Salix babylonica L., Schinus terebinthifolius Raddi, Senna macranthera (DC. Ex Collad.) H. S. Irwin \& Barneby, Senna multijuga (LC Rich) Irwin et Barneby, Syzygium cumini (L.) Skeels and Tipuana tipu (Benth) Kuntze.

In each of these species' trees, five samples from vegetative branches were collected, at the positions North, South, East and West of the crown, taken in the middle and top center parts. For the analyses, young and ageing leaves were excluded, only the mature leaves were considered.

The collected leaves were packaged in paper bags that were identified and forwarded to the Forest Ecology Laboratory, of the Federal University of Santa Maria (UFSM). There, they went through a decontamination process, being washed with distilled water and neutral detergent in the concentration of $0.1 \%$ during 02 minutes, in order to avoid nutrient leaching, according to methodology developed by Silva (1999).

After the decontamination process, the leaves samples were dried out in a kiln of circulation and air renovation in a temperature of $65^{\circ} \mathrm{C}$ until they reached constant weight. Then, they were crushed and analyzed to determine the levels of the following micronutrients: Boron (B), Copper $(\mathrm{Cu})$, Iron $(\mathrm{Fe})$, Manganese $(\mathrm{Mn})$ and Zinc $(\mathrm{Zn})$, following the methodology proposed by Tedesco et al. (1995).

Regarding the criteria for the classes' limits, ranges of micronutrients contents were determined for each analysed nutrient per species, adapted from Lúcio et al. (1999). The ranges were as follows: very low $(<-2 \mathrm{~s})$; low $(-2 \mathrm{~s} x-1 \mathrm{~s})$, medium $(-1 \mathrm{~s} \mathrm{x}+1 \mathrm{~s})$; high $(+1 \mathrm{~s} x+2 \mathrm{~s})$ and very high $(>2 \mathrm{~s})$, where " $\mathrm{x}$ " represents the mean and " $\mathrm{s}$ " the standard deviation.
After getting all of the micronutrients levels from the leaves samples from every tree individuals of each studied species, the mean and standard deviation of each nutrient were estimated. For statistical analyses, the homogeneity of variance of the data was verified by the Bartlett test and the normality of distribution was verified by the Lilliefors test. All of the variables were modified into $\log (\mathrm{x})$.

In addition, the Skott-Knott test, in terms of $5 \%$ of error probability, was utilized for the means contrasts. All of the statistical analyses were conducted within the software Assistat v. 7.7 beta (Silva 2014).

\section{RESULTS}

Table I shows the micronutrients contents observed in the leaves of the 23 tree species present in the neighborhood sampled, in the city of Santa Maria - RS. The results reveals a statistical difference for all of the micronutrients levels.

Table II presents the nutritional limits for the micronutrients levels of the tree species utilized in the street afforestation in the South Region of Brazil, providing better conditions to evaluate results from laboratorial analyses and to make decisions regarding nutritional recommendations for tree species used in urban forestry. According to Lúcio et al. (2010) and Brun et al. (2012), through this nutritional analysis, it is possible to verify if the values are higher or lower than the nutritional limits.

The achieved results of the micronutrients contents for the arboreal species utilized in urban arboriculture were compared to the considered adequate foliar concentration of nutrients for tree species, obtained from studies conducted by Malavolta et al. (1997), Larcher (2000), Silveira et al. (2005) and Dechen and Nachtigall (2006). According to these authors, the appropriate levels of micronutrients $\left(\mathrm{mg} \mathrm{kg}^{-1}\right)$ are the following: B 
TABLE I

Number of trees (n), mean (x) and standard deviation (SD) of the foliar concentrations of micronutrients in tree species utilized in street afforestation - Rio Grande do Sul State - Brazil.

\begin{tabular}{|c|c|c|c|c|c|c|c|}
\hline \multirow{2}{*}{ Species } & \multirow{2}{*}{ n. } & & B & $\mathbf{C u}$ & $\mathbf{F e}$ & Mn & $\mathbf{Z n}$ \\
\hline & & & \multicolumn{5}{|c|}{$\mathrm{mg} \mathrm{kg}^{-1}$} \\
\hline \multirow{2}{*}{ Campomanesia xanthocarpa } & \multirow{2}{*}{9} & $\mathrm{x}$ & $37.78 \mathrm{a}$ & $6.60 \mathrm{c}$ & $72.04 \mathrm{~d}$ & $83.85 \mathrm{~b}$ & $24.36 \mathrm{a}$ \\
\hline & & SD & 16.67 & 1.68 & 25.63 & 45.80 & 7.23 \\
\hline \multirow{2}{*}{ Carya illinoensis } & \multirow{2}{*}{5} & $\mathrm{x}$ & $28.96 \mathrm{~b}$ & $7.38 \mathrm{c}$ & $69.33 \mathrm{~d}$ & $446.59 \mathrm{a}$ & $33.47 \mathrm{a}$ \\
\hline & & SD & 6.51 & 3.20 & 29.15 & 315.70 & 15.25 \\
\hline \multirow{2}{*}{ Eriobotrya japonica } & \multirow{2}{*}{7} & $\mathrm{x}$ & $21.95 \mathrm{~b}$ & $6.06 \mathrm{c}$ & $177.42 \mathrm{a}$ & $49.91 \mathrm{c}$ & $36.03 \mathrm{a}$ \\
\hline & & SD & 4.78 & 1.87 & 67.38 & 20.10 & 12.68 \\
\hline \multirow{2}{*}{ Eugenia involucrata } & \multirow{2}{*}{6} & $\mathrm{x}$ & $51.58 \mathrm{a}$ & $6.99 \mathrm{c}$ & $68.45 \mathrm{~d}$ & $25.85 \mathrm{c}$ & $16.82 \mathrm{~b}$ \\
\hline & & SD & 19.63 & 2.52 & 13.72 & 10.48 & 7.47 \\
\hline \multirow{2}{*}{ Eugenia uniflora } & \multirow{2}{*}{10} & $\mathrm{x}$ & $46.37 \mathrm{a}$ & $5.09 \mathrm{~d}$ & $82.23 \mathrm{~d}$ & $32.41 \mathrm{c}$ & $29.27 \mathrm{a}$ \\
\hline & & SD & 13.59 & 2.37 & 28.84 & 16.63 & 18.79 \\
\hline \multirow{2}{*}{ Grevillea robusta } & \multirow{2}{*}{7} & $\mathrm{x}$ & $11.95 \mathrm{c}$ & $4.48 \mathrm{~d}$ & $106.08 \mathrm{~b}$ & $144.15 \mathrm{a}$ & $12.77 \mathrm{~b}$ \\
\hline & & SD & 2.26 & 2.05 & 32.93 & 99.97 & 8.87 \\
\hline \multirow{2}{*}{ Handroanthus chrysotrichus } & \multirow{2}{*}{10} & $\mathrm{x}$ & $66.44 \mathrm{a}$ & $10.04 \mathrm{~b}$ & $157.10 \mathrm{~b}$ & $347.23 \mathrm{a}$ & $28.45 \mathrm{a}$ \\
\hline & & SD & 22.14 & 2.73 & 67.52 & 180.12 & 6.33 \\
\hline \multirow{2}{*}{ Handroanthus heptaphyllus } & \multirow{2}{*}{9} & $\mathrm{x}$ & $47.44 \mathrm{a}$ & $7.89 \mathrm{c}$ & $110.65 \mathrm{c}$ & $43.65 \mathrm{c}$ & $18.03 \mathrm{~b}$ \\
\hline & & SD & 14.83 & 1.49 & 61.10 & 29.43 & 8.20 \\
\hline \multirow{2}{*}{ Inga marginata } & \multirow{2}{*}{9} & $\mathrm{x}$ & $25.32 \mathrm{~b}$ & $7.73 \mathrm{c}$ & $86.32 \mathrm{c}$ & $85.70 \mathrm{~b}$ & $23.41 \mathrm{a}$ \\
\hline & & $\mathrm{SD}$ & 9.54 & 2.44 & 36.43 & 44.77 & 5.69 \\
\hline \multirow{2}{*}{ Jacaranda mimosifolia } & \multirow{2}{*}{7} & $\mathrm{x}$ & $21.38 \mathrm{~b}$ & $8.94 \mathrm{c}$ & $137.71 \mathrm{~b}$ & $24.99 \mathrm{c}$ & $18.99 \mathrm{~b}$ \\
\hline & & $\mathrm{SD}$ & 3.52 & 3.17 & 46.57 & 15.52 & 9.46 \\
\hline \multirow{2}{*}{ Lagerstroemia indica } & \multirow{2}{*}{15} & $\mathrm{x}$ & $41.61 \mathrm{a}$ & $14.56 \mathrm{a}$ & $133.88 \mathrm{~b}$ & $163.43 \mathrm{~b}$ & $43.77 \mathrm{a}$ \\
\hline & & SD & 12.90 & 7.83 & 91.07 & 124.27 & 21.89 \\
\hline \multirow{2}{*}{ Melia azedarach } & 15 & $\mathrm{x}$ & $37.36 \mathrm{a}$ & $6.43 \mathrm{~d}$ & $108.79 \mathrm{c}$ & $30.04 \mathrm{c}$ & $20.46 \mathrm{~b}$ \\
\hline & 15 & SD & 9.93 & 3.82 & 38.30 & 10.07 & 6.22 \\
\hline Morus niorg & 6 & $\mathrm{x}$ & $69.99 \mathrm{a}$ & $5.38 \mathrm{~d}$ & $100.78 \mathrm{c}$ & $88.48 \mathrm{~b}$ & $29.33 \mathrm{a}$ \\
\hline & 0 & SD & 28.17 & 1.94 & 34.58 & 48.68 & 7.64 \\
\hline Pelthonhorum duhium & 6 & $\mathrm{x}$ & $32.71 \mathrm{~b}$ & $6.69 \mathrm{c}$ & $93.46 \mathrm{c}$ & $137.59 \mathrm{~b}$ & $19.35 \mathrm{~b}$ \\
\hline Pelthopnorum аивıтm & 0 & SD & 17.53 & 2.44 & 31.77 & 66.14 & 3.69 \\
\hline Poincianella nluviosa & 11 & $\mathrm{x}$ & $36.10 \mathrm{a}$ & $7.28 \mathrm{c}$ & $113.49 \mathrm{~b}$ & $65.63 \mathrm{c}$ & $13.23 \mathrm{~b}$ \\
\hline Poinclanella piuviosa & 11 & SD & 12.34 & 1.80 & 23.26 & 49.01 & 5.63 \\
\hline Psidium cattleyanum & 7 & $\mathrm{x}$ & $29.64 \mathrm{~b}$ & $8.57 \mathrm{c}$ & $52.77 \mathrm{~d}$ & $100.16 \mathrm{~b}$ & $15.78 \mathrm{~b}$ \\
\hline г stalum camteуаны & 1 & SD & 9.50 & 4.15 & 15.73 & 32.48 & 12.44 \\
\hline Psidium ougiaya & 8 & $\mathrm{x}$ & $45.64 \mathrm{a}$ & $13.12 \mathrm{~b}$ & $105.61 \mathrm{c}$ & $72.37 \mathrm{c}$ & $23.14 \mathrm{a}$ \\
\hline Р staium gиајауа & 0 & SD & 9.02 & 4.85 & 51.78 & 29.08 & 8.44 \\
\hline Salix habvlonica & 6 & $\mathrm{x}$ & $27.95 \mathrm{~b}$ & $6.60 \mathrm{c}$ & $184.02 \mathrm{a}$ & $115.54 \mathrm{~b}$ & $42.47 \mathrm{a}$ \\
\hline saix равугоnica & 0 & SD & 5.46 & 1.90 & 53.82 & 73.65 & 32.03 \\
\hline
\end{tabular}


TABLE I (continuation)

\begin{tabular}{|c|c|c|c|c|c|c|c|}
\hline \multirow{2}{*}{ Species } & \multirow{2}{*}{ n. } & & B & $\mathrm{Cu}$ & $\mathrm{Fe}$ & Mn & Zn \\
\hline & & & \multicolumn{5}{|c|}{$\mathrm{mg} \mathrm{kg}^{-1}$} \\
\hline \multirow{2}{*}{ Schinus terebinthifolius } & \multirow{2}{*}{6} & $\mathrm{x}$ & $35.53 \mathrm{a}$ & $7.34 \mathrm{c}$ & $86.15 \mathrm{c}$ & $51.39 \mathrm{c}$ & $17.99 \mathrm{~b}$ \\
\hline & & SD & 18.57 & 5.64 & 22.87 & 20.35 & 12.26 \\
\hline \multirow{2}{*}{ Senna macranthera } & \multirow{2}{*}{6} & $\mathrm{x}$ & $43.75 \mathrm{a}$ & $5.91 \mathrm{c}$ & $94.11 \mathrm{c}$ & $42.77 \mathrm{c}$ & $15.79 \mathrm{~b}$ \\
\hline & & SD & 12.95 & 0.87 & 11.36 & 13.72 & 6.19 \\
\hline \multirow{2}{*}{ Senna multijuga } & \multirow{2}{*}{6} & $\mathrm{x}$ & $21.05 \mathrm{~b}$ & $6.11 \mathrm{c}$ & $78.06 \mathrm{c}$ & $84.49 \mathrm{~b}$ & $16.46 \mathrm{~b}$ \\
\hline & & SD & 5.05 & 1.83 & 14.54 & 39.09 & 4.43 \\
\hline \multirow{2}{*}{ Syzygium cumini } & \multirow{2}{*}{7} & $\mathrm{x}$ & $31.17 \mathrm{~b}$ & $4.46 \mathrm{~d}$ & $69.84 \mathrm{~d}$ & $79.14 b$ & $9.51 \mathrm{~b}$ \\
\hline & & SD & 9.36 & 1.71 & 13.69 & 29.29 & 1.30 \\
\hline \multirow{2}{*}{ Tipuana tipu } & \multirow{2}{*}{10} & $\mathrm{x}$ & $21.07 \mathrm{~b}$ & $5.79 \mathrm{~d}$ & $111.58 \mathrm{c}$ & $47.87 \mathrm{c}$ & $20.96 \mathrm{a}$ \\
\hline & & SD & 6.27 & 3.09 & 28.50 & 27.34 & 7.34 \\
\hline- & - & $\mathrm{CV}(\%)$ & 9.81 & 20.56 & 6.58 & 13.58 & 13.53 \\
\hline
\end{tabular}

Note: Numbers followed by the same letter in the column are not significantly different $(\mathrm{p} \leq 0.05)$.

TABLE II

Foliar nutritional limits to arboreal species used in the urban forestry of the Santa Maria City - Rio Grande do Sul State - Brazil.

\begin{tabular}{|c|c|c|c|c|c|c|}
\hline \multirow{2}{*}{ Species } & \multirow{2}{*}{ Limits } & B & $\mathrm{Cu}$ & $\mathrm{Fe}$ & Mn & Zn \\
\hline & & \multicolumn{5}{|c|}{$\mathrm{mg} \mathrm{kg}^{-1}$} \\
\hline \multirow{5}{*}{$\begin{array}{c}\text { Campomanesia } \\
\text { xanthocarpa }\end{array}$} & VL & $<4.45$ & $<3.25$ & $<20.79$ & $0.0^{*}$ & $<9.90$ \\
\hline & $\mathrm{L}$ & $4.45-21.11$ & $3.25-4.92$ & $20.79-46.42$ & $0.0-38.05$ & $9.90-17.13$ \\
\hline & M & $21.11-54.45$ & $4.92-8.28$ & $46.42-97.67$ & $38.05-129.65$ & $17.13-31.60$ \\
\hline & $\mathrm{H}$ & $54.45-71.12$ & $8.28-9.96$ & $97.67-123.30$ & $129.65-175.44$ & $31.60-38.83$ \\
\hline & $\mathrm{VH}$ & $>71.12$ & $>9.96$ & $>123.30$ & $>175.44$ & $>38.83$ \\
\hline \multirow{5}{*}{ Carya illinoensis } & VL & $<15.93$ & $<0.98$ & $<11.02$ & 0.0 & $<2.96$ \\
\hline & $\mathrm{L}$ & $15.93-22.45$ & $0.98-4.18$ & $11.02-40.17$ & $0.0-130.89$ & $2.96-18.22$ \\
\hline & M & $22.45-35.47$ & $4.18-10.58$ & $40.17-98.48$ & $130.89-762.28$ & $18.22-48.72$ \\
\hline & $\mathrm{H}$ & $35.47-41.99$ & $10.58-13.77$ & $98.48-127.63$ & $762.28-1077.98$ & $48.72-63.98$ \\
\hline & $\mathrm{VH}$ & $>41.99$ & $>13.77$ & $>127.63$ & $>1077.98$ & $>63.98$ \\
\hline \multirow{5}{*}{ Eriobotrya japonica } & VL & $<12.39$ & $<2.31$ & $<42.65$ & $<9.71$ & $<10.67$ \\
\hline & $\mathrm{L}$ & $12.39-17.17$ & $2.31-4.18$ & $42.65-110.03$ & $9.71-29.81$ & $10.67-23.35$ \\
\hline & M & $17.17-26.74$ & $4.18-7.93$ & $110.03-244.80$ & $29.81-70.01$ & $23.35-48.71$ \\
\hline & $\mathrm{H}$ & $26.74-31.52$ & $7.93-9.80$ & $244.80-312.19$ & $70.01-90.11$ & 48.71-61.39 \\
\hline & $\mathrm{VH}$ & $>31.52$ & $>9.80$ & $>312.19$ & $>90.11$ & $>61.39$ \\
\hline \multirow{5}{*}{ Eugenia involucrata } & VL & $<12.32$ & $<1.94$ & $<41.01$ & $<4.89$ & $<1.87$ \\
\hline & $\mathrm{L}$ & $12.32-31.95$ & $1.94-4.46$ & $41.01-54.73$ & $4.89-15.37$ & $1.87-9.35$ \\
\hline & M & $31.95-71.21$ & $4.46-9.51$ & $54.73-82.17$ & $15.37-36.33$ & $9.35-24.29$ \\
\hline & $\mathrm{H}$ & $71.21-90.84$ & $9.51-12.03$ & $82.17-95.89$ & $36.33-46.81$ & $24.29-31.77$ \\
\hline & VH & $>90.84$ & $>12.03$ & $>95.89$ & $>46.81$ & $>31.77$ \\
\hline
\end{tabular}


TABLE II (continuation)

\begin{tabular}{|c|c|c|c|c|c|c|}
\hline \multirow{2}{*}{ Species } & \multirow{2}{*}{ Limits } & B & $\mathrm{Cu}$ & $\mathrm{Fe}$ & Mn & $\mathbf{Z n}$ \\
\hline & & \multicolumn{5}{|c|}{$\mathrm{mg} \mathrm{kg}^{-1}$} \\
\hline \multirow{5}{*}{ Eugenia uniflora } & VL & $<19.19$ & $<0.35$ & $<24.54$ & 0.0 & 0.0 \\
\hline & $\mathrm{L}$ & $19.19-32.78$ & $0.35-2.72$ & $24.54-53.38$ & $0.0-15.77$ & $0.0-10.48$ \\
\hline & M & $32.78-59.97$ & $2.72-7.46$ & $53.38-111.07$ & $15.77-49.04$ & $10.48-48.06$ \\
\hline & $\mathrm{H}$ & $59.97-73.56$ & $7.46-9.83$ & $111.07-139.91$ & $49.04-65.67$ & $48.06-66.85$ \\
\hline & VH & $>73.56$ & $>9.83$ & $>139.91$ & $>65.67$ & $>66.85$ \\
\hline \multirow{5}{*}{ Grevillea robusta } & VL & $<7.43$ & $<0.37$ & $<40.23$ & 0.0 & 0.0 \\
\hline & $\mathrm{L}$ & $7.43-9.69$ & $0.37-2.42$ & $40.23-73.15$ & $0.0-44.18$ & $0.0-3.89$ \\
\hline & M & $9.69-14.21$ & $7.42-6.53$ & $73.15-139.01$ & $44.18-244.13$ & $3.89-21.64$ \\
\hline & $\mathrm{H}$ & $14.21-16.47$ & $6.53-8.59$ & 139.01-171.94 & $244.13-344.10$ & $21.64-30.51$ \\
\hline & $\mathrm{VH}$ & $>16.47$ & $>8.59$ & $>171.94$ & $>344.10$ & $>30.51$ \\
\hline \multirow{5}{*}{$\begin{array}{l}\text { Handroanthus } \\
\text { chrysotrichus }\end{array}$} & $\mathrm{VL}$ & $<22.17$ & $<4.58$ & $<22.05$ & 0.0 & $<15.79$ \\
\hline & $\mathrm{L}$ & $22.17-44.30$ & $4.58-7.31$ & $22.05-89.58$ & $0.0-167.11$ & $15.79-22.12$ \\
\hline & M & $44.30-88.58$ & $7.31-12.77$ & $89.58-224.63$ & $167.11-527.35$ & $22.12-34.79$ \\
\hline & $\mathrm{H}$ & $88.58-110.71$ & $12.77-15.50$ & $224.63-292.15$ & $527.35-707.47$ & $34.79-41.12$ \\
\hline & VH & $>110.71$ & $>15.50$ & $>292.15$ & $>707.47$ & $>41.12$ \\
\hline \multirow{5}{*}{ Handroanthus heptaphyllus } & $\mathrm{VL}$ & $<17.78$ & $<4.91$ & 0.0 & 0.0 & $<1.63$ \\
\hline & $\mathrm{L}$ & $17.78-32.61$ & $4.91-6.40$ & $0.0-49.55$ & $0.0-14.22$ & $1.63-9.83$ \\
\hline & M & $32.61-62.26$ & $6.40-9.38$ & $49.55-171.75$ & $14.22-73.08$ & $9.83-26.23$ \\
\hline & $\mathrm{H}$ & $62.26-77.09$ & $9.38-10.87$ & $171.75-232.84$ & $73.08-102.51$ & $26.23-34.44$ \\
\hline & $\mathrm{VH}$ & $>77.09$ & $>10.87$ & $>232.84$ & $>102.51$ & $>34.44$ \\
\hline \multirow{5}{*}{ Inga marginata } & $\mathrm{VL}$ & $<6.24$ & $<2.85$ & $<13.46$ & 0.0 & $<12.03$ \\
\hline & $\mathrm{L}$ & $6.24-15.78$ & $2.85-5.29$ & $13.46-49.89$ & $0.0-40.93$ & $12.3-17.72$ \\
\hline & M & $15.78-34.85$ & $5.29-10.17$ & $49.89-122.75$ & $40.93-130.47$ & $17.72-29.10$ \\
\hline & $\mathrm{H}$ & $34.85-44.39$ & $10.17-12.62$ & $122.75-159.18$ & $130.47-175.24$ & $29.10-34.79$ \\
\hline & $\mathrm{VH}$ & $>44.39$ & $>12.62$ & $>159.18$ & $>175.24$ & $>34.79$ \\
\hline \multirow{5}{*}{ Jacaranda mimosifolia } & VL & $<14.34$ & $<2.60$ & $<44.57$ & 0.0 & $<0.08$ \\
\hline & $\mathrm{L}$ & $14.34-17.86$ & $2.60-5.77$ & $44.57-91.14$ & $0.0-9.47$ & $0.08-9.54$ \\
\hline & M & $17.86-24.90$ & $5.77-12.11$ & $91.14-184.27$ & $9.47-40.51$ & $9.54-28.45$ \\
\hline & $\mathrm{H}$ & $24.90-28.42$ & $12.11-15.29$ & $184.27-230.84$ & $40.51-56.03$ & $28.45-37.90$ \\
\hline & VH & $>28.42$ & $>15.29$ & $>230.84$ & $>56.03$ & $>37.90$ \\
\hline \multirow{5}{*}{ Lagerstroemia indica } & $\mathrm{VL}$ & $<15.80$ & 0.0 & 0.0 & 0.0 & $<-0.01$ \\
\hline & $\mathrm{L}$ & $15.80-28.71$ & $0.0-6.73$ & $0.0-42.81$ & $0.0-39.15$ & $-0.01-21.88$ \\
\hline & M & $28.71-54.52$ & $6.73-22.39$ & $42.81-224.95$ & $39.15-287.70$ & $21.88-65.65$ \\
\hline & $\mathrm{H}$ & $54.52-67.42$ & $22.39-30.22$ & $224.95-316.02$ & $287.70-411.97$ & $65.65-87.54$ \\
\hline & VH & $>67.42$ & $>30.22$ & $>316.02$ & $>411.97$ & $>87.54$ \\
\hline
\end{tabular}


TABLE II (continuation)

\begin{tabular}{|c|c|c|c|c|c|c|}
\hline \multirow{2}{*}{ Species } & \multirow{2}{*}{ Limits } & B & $\mathrm{Cu}$ & $\mathrm{Fe}$ & Mn & $\mathbf{Z n}$ \\
\hline & & \multicolumn{5}{|c|}{$\mathrm{mg} \mathrm{kg}^{-1}$} \\
\hline \multirow{5}{*}{ Melia azedarach } & VL & $<17.50$ & 0.0 & $<32.20$ & $<9.91$ & $<8.01$ \\
\hline & $\mathrm{L}$ & $17.50-27.43$ & $0.0-2.61$ & $32.20-70.49$ & $9.91-19.98$ & $8.01-14.23$ \\
\hline & M & $27.43-47.28$ & $2.61-10.24$ & $70.49-147.08$ & $19.98-40.11$ & $14.23-26.68$ \\
\hline & $\mathrm{H}$ & $47.28-57.21$ & $10.25-14.07$ & $147.08-185.38$ & $40.11-50.18$ & $26.68-32.90$ \\
\hline & $\mathrm{VH}$ & $>57.21$ & $>14.07$ & $>185.38$ & $>50.18$ & $>32.90$ \\
\hline \multirow{5}{*}{ Morus nigra } & VL & $<13.64$ & $<1.50$ & $<31.61$ & 0.0 & $<14.06$ \\
\hline & $\mathrm{L}$ & $13.64-41.81$ & $1.50-3.44$ & $31.61-66.19$ & $0.0-39.80$ & $14.06-21.69$ \\
\hline & M & $41.81-98.16$ & $3.44-7.31$ & $66.19-135.36$ & $39.80-137.15$ & $21.69-36.97$ \\
\hline & $\mathrm{H}$ & $98.16-126.33$ & $7.31-9.25$ & $135.36-169.94$ & $137.15-185.83$ & $36.97-44.61$ \\
\hline & VH & $>126.33$ & $>9.25$ & $>169.94$ & $>185.83$ & $>44.61$ \\
\hline \multirow{5}{*}{ Peltophorum dubium } & VL & $<-2.34$ & $<1.81$ & $<29.92$ & $<5.32$ & $<11.97$ \\
\hline & $\mathrm{L}$ & $-2.34-15.18$ & $1.81-4.25$ & $29.92-61.69$ & $5.32-71.46$ & $11.97-15.66$ \\
\hline & M & $15.18-50.23$ & $4.25-9.12$ & $61.69-125.23$ & $71.46-203.73$ & $15.66-23.03$ \\
\hline & $\mathrm{H}$ & $50.23-67.76$ & $9.12-11.56$ & $125.23-157.00$ & $203.73-269.87$ & $23.03-26.72$ \\
\hline & $\mathrm{VH}$ & $>67.76$ & $>11.56$ & $>157.00$ & $>269.87$ & $>26.72$ \\
\hline \multirow{5}{*}{ Poincianella pluviosa } & VL & $<11.43$ & $<3.67$ & $<66.96$ & 0.0 & $<1.97$ \\
\hline & $\mathrm{L}$ & $11.43-23.77$ & $3.67-5.48$ & $66.96-90.22$ & $0.0-16.62$ & $1.97-7.60$ \\
\hline & M & $23.77-48.44$ & $5.48-9.08$ & $90.22-136.75$ & $16.62-114.63$ & $7.60-18.86$ \\
\hline & $\mathrm{H}$ & $48.44-60.77$ & $9.08-10.89$ & $136.75-160.02$ & $114.63-163.64$ & $18.86-24.49$ \\
\hline & VH & $>60.77$ & $>10.89$ & $>160.02$ & $>163.64$ & $>24.49$ \\
\hline \multirow{5}{*}{ Psidium cattleyanum } & VL & $<10.65$ & $<0.28$ & $<21.30$ & $<35.19$ & 0.0 \\
\hline & $\mathrm{L}$ & $10.65-20.14$ & $0.28-4.43$ & $21.30-37.04$ & $35.19-67.67$ & $0.0-3.34$ \\
\hline & M & $20.14-39.14$ & $4.43-12.72$ & $37.04-68.51$ & $67.67-132.64$ & $3.34-28.22$ \\
\hline & $\mathrm{H}$ & $39.14-48.63$ & $12.72-16.87$ & $68.51-84.24$ & $132.64-165.12$ & $28.22-40.66$ \\
\hline & $\mathrm{VH}$ & $>48.63$ & $>16.87$ & $>84.24$ & $>165.12$ & $>40.66$ \\
\hline \multirow{5}{*}{ Psidium guajava } & VL & $<27.60$ & $<3.42$ & $<2.06$ & $<14.22$ & $<6.26$ \\
\hline & $\mathrm{L}$ & $27.60-36.62$ & $3.42-8.27$ & $2.06-53.84$ & $14.22-43.30$ & $6.26-14.70$ \\
\hline & M & $36.62-54.66$ & $8.27-17.98$ & $53.84-157.39$ & $43.30-101.45$ & $14.70-31.57$ \\
\hline & $\mathrm{H}$ & $54.66-63.68$ & $17.98-22.83$ & $157.39-209.17$ & $101.45-130.52$ & $31.57-40.01$ \\
\hline & VH & $>63.68$ & $>22.83$ & $>209.17$ & $>130.52$ & $>40.01$ \\
\hline \multirow{5}{*}{ Salix babylonica } & VL & $<17.03$ & $<2.79$ & $<76.38$ & 0.0 & 0.0 \\
\hline & $\mathrm{L}$ & $17.03-22.49$ & $2.79-4.70$ & $76.38-130.20$ & $0.0-41.89$ & $0.0-10.44$ \\
\hline & M & $22.49-33.40$ & $4.70-8.50$ & $130.20-237.84$ & $41.89-189.19$ & $10.44-74.50$ \\
\hline & $\mathrm{H}$ & $33.40-38.86$ & $8.50-10.40$ & $237.84-291.66$ & $189.19-262.85$ & $74.50-106.53$ \\
\hline & VH & $>38.86$ & $>10.40$ & $>291.66$ & $>262.85$ & $>106.53$ \\
\hline
\end{tabular}


TABLE II (continuation)

\begin{tabular}{|c|c|c|c|c|c|c|}
\hline \multirow{2}{*}{ Species } & \multirow{2}{*}{ Limits } & B & $\mathrm{Cu}$ & $\mathrm{Fe}$ & Mn & Zn \\
\hline & & \multicolumn{5}{|c|}{$\mathrm{mg} \mathrm{kg}^{-1}$} \\
\hline \multirow{5}{*}{ Schinus terebinthifolius } & VL & 0.0 & 0.0 & $<40.40$ & $<10.68$ & 0.0 \\
\hline & $\mathrm{L}$ & $0.0-16.96$ & $0.0-1.70$ & $40.40-63.27$ & $10.68-31.04$ & $0.0-5.73$ \\
\hline & M & $16.96-54.09$ & $1.70-12.98$ & $63.27-109.02$ & $31.04-71.74$ & $5.73-30.26$ \\
\hline & $\mathrm{H}$ & $54.10-72.66$ & $12.98-18.62$ & $109.02-131.89$ & $71.74-92.10$ & $30.26-42.52$ \\
\hline & $\mathrm{VH}$ & $>72.66$ & $>18.62$ & $>131.89$ & $>92.10$ & $>42.52$ \\
\hline \multirow{5}{*}{ Senna macranthera } & VL & $<17.86$ & $<4.17$ & $<71.39$ & $<15.33$ & $<3.42$ \\
\hline & $\mathrm{L}$ & $17.86-30.81$ & $4.17-5.04$ & $71.39-82.75$ & $15.33-29.05$ & $3.42-9.60$ \\
\hline & M & $30.81-56.70$ & $5.04-6.78$ & $82.75-105.47$ & $29.05-56.48$ & $9.60-21.98$ \\
\hline & $\mathrm{H}$ & $56.70-69.64$ & $6.78-7.65$ & $105.47-116.83$ & $56.48-70.20$ & $21.98-28.16$ \\
\hline & $\mathrm{VH}$ & $>69.64$ & $>7.65$ & $>116.83$ & $>70.20$ & $>28.16$ \\
\hline \multirow{5}{*}{ Senna multijuga } & VL & $<10.95$ & $<2.45$ & $<48.98$ & $<6.31$ & $<7.59$ \\
\hline & $\mathrm{L}$ & $10.95-16.00$ & $2.45-4.28$ & $48.98-63.52$ & $6.31-45.40$ & $7.59-12.02$ \\
\hline & M & $16.00-26.10$ & $4.28-7.94$ & $63.52-92.60$ & $45.40-123.58$ & $12.02-20.89$ \\
\hline & $\mathrm{H}$ & $26.10-31.14$ & $7.94-31.14$ & $92.60-107.14$ & $123.58-162.66$ & $20.89-25.32$ \\
\hline & $\mathrm{VH}$ & $>31.14$ & $>9.77$ & $>107.14$ & $>162.66$ & $>25.32$ \\
\hline \multirow{5}{*}{ Syzygium cumini } & VL & $<12.46$ & $<1.05$ & $<42.46$ & $<20.56$ & $<6.90$ \\
\hline & $\mathrm{L}$ & $12.46-21.81$ & $1.05-2.75$ & $42.46-56.15$ & $20.56-49.85$ & $6.90-8.21$ \\
\hline & M & $21.81-40.53$ & $2.75-6.17$ & $56.15-83.52$ & $49.85-108.43$ & $8.21-10.81$ \\
\hline & $\mathrm{H}$ & $40.53-49.88$ & $6.17-7.87$ & $83.52-97.21$ & $108.43-137.72$ & $10.81-12.12$ \\
\hline & $\mathrm{VH}$ & $>49.88$ & $>7.87$ & $>97.21$ & $>137.72$ & $>12.12$ \\
\hline \multirow{5}{*}{ Tipuana tipu } & VL & $<8.53$ & 0.0 & $<54.57$ & 0.0 & $<6.28$ \\
\hline & $\mathrm{L}$ & $8.53-14.80$ & $0.0-2.69$ & $54.57-83.07$ & $0.0-20.53$ & $6.28-13.62$ \\
\hline & M & $14.80-27.34$ & $2.69-8.88$ & $83.07-140.08$ & $20.53-75.20$ & $13.62-28.30$ \\
\hline & $\mathrm{H}$ & $27.34-33.61$ & $8.88-11.97$ & $140.08-168.58$ & $75.20-102.54$ & $28.30-35.65$ \\
\hline & VH & $>33.61$ & $>11.97$ & $>168.58$ & $>102.54$ & $>35.65$ \\
\hline
\end{tabular}

Abbreviations: VL: Very low; L: Low; M: Middle; H: High; VH: Very High; * in these cases, where there are zero $(0.0)$ values, they occur due to the high variation (high standard deviation), resulting in a negative value in the calculation of the class limit, which does not represent the reality, being assigned with zero value.

(20.0 to 60.0); $\mathrm{Cu}$ (5.0 to 20.0); $\mathrm{Fe}$ (50.0 to 150.0); Mn (190.0 to 840.0); Zn (20.0 to 50.0).

It was noted that, for $G$. robusta, the concentration of B was lower than the level considered adequate and for $H$. chrysotrichus e $M$. nigra, the levels of the same nutrient were higher than the recommended interval. The contents of $\mathrm{Cu}$ were lower than the adequate level for G. robusta e S. cumini. The concentration of Fe reached a high level for E. japonica, H. chrysotrichus and $S$. babylonica.

For C.xanthocarpa, E.japonica, E. involucrata, E. uniflora, G. robusta, H. heptaphyllus, I. marginata, J. mimosifolia, L. indica, M. azedarach, M. nigra, P. dubium, P. pluviosa, P. cattleyanum, P. guajava, S. babylonica, S. terebinthifolius, $S$. macranthera, S. multijuga, S. cumini and T. tipu, the contents of Mn were under the level considered adequate. The levels of $\mathrm{Mn}$ were inside the range 
designed as satisfactory only for C. illinoensis and $H$. chrysotrichus. The concentration levels of $\mathrm{Zn}$ were low for E. involucrata, G. robusta, $H$. heptaphyllus, J. mimosaefolia, P. dubium, $P$. pluviosa, P. cattleyanum, $S$. terebinthifolius, $S$. macranthera, S. multijuga and S. cumini.

\section{DISCUSSION}

Micronutrients are needed in smaller quantities and may still be toxic in high amounts. However, the deficiency of a given micronutrient can have serious adverse effects on tree health (Lilly 2015). In order for these more serious adverse effects to occur, which may compromise the tree's landscape functions, especially at the level of deficiencies, nutrient contents should be classified as priority VL (very low) (Table II).

The foliar contents of B were interpreted based on what was acknowledged by Malavolta et al. (1997), Larcher (2000) and Dechen and Nachtigall (2006) and others authors who pointed out that a concentration between 20.0 to $60.0 \mathrm{mg}$ $\mathrm{kg}^{-1}$ would be important to show that the plant is well-nourished by the nutrient. Based on this information, the concentration of $\mathrm{B}$ reached a high level for the species $H$. chrysotrichus and $M$. nigra and low level for G. robusta.

The element $\mathrm{B}$ is an essential micronutrient for plants, and its symptoms of lack appears firstly in young plants since it is an immobile/poorly mobile nutrient in vegetable tissues. Raven et al. (1992) assert that one of the main functions of Boron is to influence the utilization of $\mathrm{Ca}^{2+}$, the synthesis of nucleic acids and the cell membrane integrity. Due to the fact that the foliar concentrations of the species focused in this study showed elevated contents of $\mathrm{Ca}$ in areas where there is an increased supply of $\mathrm{Ca}$, one of the greater functions of $\mathrm{B}$ would be already completed, indicating that the plants would not demand to absorb this element anymore.
In agreement with interpretation parameters expressed by Malavolta et al. (1997), Larcher (2000) and Dechen and Nachtigall (2006), the B reported deficiency only for G. robusta. Despite the result, the foliar concentration of this element varies greatly for forest species (Coelho and Verlengia 1973, Brun et al. 2012).

For Brun et al. (2012), the levels of B in leaves of Cedrella fissilis and P. dubium were 16.89 and $15.44 \mathrm{mg} \mathrm{kg}^{-1}$, indicating a slight deficiency of this nutrient. Authors as Lúcio et al. (2010), inspecting limits of precaution and control in nutritional analyses of Acacia mearnsii, Eucalyptus spp. and Pinus taeda, observed values for B as $32.39,33.86$ and $18.27 \mathrm{mg} \mathrm{kg}^{-1}$, respectively, rates that can be implied as adequate for A. mearnsii e Eucalyptus spp. and, for $P$. taeda, considered lower than the adequate limit, when compared to the limits recommended in the present study.

The symptoms of boron toxicity are not well known. However, boron deficiency in trees has, according to Marschner (1995) and Epstein and Bloom (2005), shortening of the internodes, intercostal chlorosis of the younger leaves, deformation of the buds and leaf blade, presence of cracks, roughness, protrusions and spots on the stem, reduction of flower formation and low rate of fruit formation, as well as deformation. A tree with these symptoms becomes, in landscape terms, derogatory of the environment in which it is.

Referring to $\mathrm{Cu}$, Dechen and Nachtigall (2006) specify that levels below $5.0 \mathrm{mg} \mathrm{kg}^{-1}$ can be assumed as lower than the desired concentration for the appropriate development of plants. The $\mathrm{Cu}$ showed a value below the adequate level for G. robusta $\left(4.48 \mathrm{mg} \mathrm{kg}^{-1}\right)$ and $S$. cumini $(4.46 \mathrm{mg}$ $\mathrm{kg}^{-1}$ ), in which both differed significantly from the other species.

In soil, the exchangeable $\mathrm{Cu}$ is absorbed by the organic matter, where the ion is fixed by humus in a more stable form than the exchangeable one. In a scenario with low content of organic matter 
and an acid pH, according to Brun et al. (2012), it is expected that the relative share of exchangeable $\mathrm{Cu}$ would be prevalent and more readily available to plants, which can explain the adequate levels for most of the species studied in this paper.

In the study carried out by Lúcio et al. (2010), the contents of $\mathrm{Cu}$ for $A$. mearnsii $\left(7.73 \mathrm{mg} \mathrm{kg}^{-1}\right)$, Eucalyptus spp. (7.13 $\mathrm{mg} \mathrm{kg}^{-1}$ ) and P. taeda (4.47 $\left.\mathrm{mg} \mathrm{kg}^{-1}\right)$, revealed to be satisfactory for this nutrient. Brun et al. (2012), evaluating the concentration levels of $\mathrm{Cu}$ in the leaves of E. uniflora $(6.29 \mathrm{mg}$ $\left.\mathrm{kg}^{-1}\right)$, Parapiptadenia rigida $\left(8.66 \mathrm{mg} \mathrm{kg}^{-1}\right), P$. pluviosa (15.75 mg kg-1), P. dubium $\left(8.34 \mathrm{mg} \mathrm{kg}^{-}\right.$ ${ }^{1}$ ) and $C$. fissilis $\left(10.06 \mathrm{mg} \mathrm{kg}^{-1}\right)$, recognized that they were within a range considered satisfactory. Those results claim that $\mathrm{Cu}$, as a nutrient, has been absorbed properly by the species.

To the species that showed $\mathrm{Cu}$ deficiency, the landscape aspects and reproduction capacity are affected, according to Epstein and Bloom (2005), Mengel and Kirkby (1987), Sorreano et al. (2012), with loss of the pollen viability, internodes decrease, the leaves become thin and twisted, with the whitish apex and the pendulum form of the trees by the reduction of the lignin synthesis. There is also leaf chlorosis or leaves with a deep blue-greenish hue, with curled edges up. Tree barks often get rough and covered in blisters, and a gum may exude from cracks in the bark.

The contents of Fe exhibited high levels for $E$. japonica (177.42 $\left.\mathrm{mg} \mathrm{kg}^{-1}\right)$, H. chrysotrichus (157.10 $\mathrm{mg} \mathrm{kg}{ }^{-1}$ ) and $S$. babylonica (184.02 $\left.\mathrm{mg} \mathrm{kg}^{-1}\right)$. As reported by Malavolta et al. (1997), Larcher (2000) and Dechen and Nachtigall (2006), the satisfactory levels of Fe to enable a decent development of tree and herbaceous plants vary from 50 to $150 \mathrm{mg} \mathrm{kg}^{-1}$ among the different species. The preferred site of occurrence of iron is in the leaves (Larcher 2000) since this element participates in the process of chrolophyll formation, electronic transportation in the photosynthesis, $\mathrm{N}_{2}$ fixation, as well as the formation of some respiratory systems of enzymes.
Caldeira et al. (2006), studying the micronutrients contents in tree species in Ombrofila Mix Mountain Forest in South of Paraná, in a condition of native forest, visualized adequate concentration of $\mathrm{Fe}$ for the species $C$. xanthocarpa $\left(96.0 \mathrm{mg} \mathrm{kg}^{-1}\right)$ and $S$. terebinthifolius $(50.0 \mathrm{mg}$ $\mathrm{kg}^{-1}$ ), similar to those found in the present study for the same specie but in urban condition $(C$. xanthocarpa, $72.04 \mathrm{mg} \mathrm{kg}^{-1}$ and $S$. terebinthifolius, $86.15 \mathrm{mg} \mathrm{kg}^{-1}$ ).

Caldeira et al. (2006) noticed high levels of Fe for Allophyllus edulis (705.67 $\mathrm{mg} \mathrm{kg}^{-1}$ ), Casearia decandra (316.35 $\mathrm{mg} \mathrm{kg}^{-1}$ ), Dicksonia sellowiana (666.67 $\mathrm{mg} \mathrm{kg}^{-1}$ ), Mimosa scabrella $(331.67 \mathrm{mg}$ $\mathrm{kg}^{-1}$ ), Myrsine umbellata (320.33 $\mathrm{mg} \mathrm{kg}^{-1}$ ), Ocotea porosa (316.33 $\left.\mathrm{mg} \mathrm{kg}^{-1}\right)$ and Symplocos uniflora (610.85 $\left.\mathrm{mg} \mathrm{kg}^{-1}\right)$. Lúcio et al. (2010) also found out high contents of Fe for A. mearnsii (166.27 mg kg $\left.{ }^{1}\right)$ and P. taeda (167.27 $\left.\mathrm{mg} \mathrm{kg}^{-1}\right)$.

The highlighted amounts of $\mathrm{Fe}$ in the leaves of E. japonica, H. chrysotrichus and S. babylonica in the urban area studied in Camobi neighborhood are a result of the species ability in absorbing the element, under extreme environmental conditions, as urban areas under pollution. As stated by Firkowski (1990), Reissmann and Biondi (1994), the intense vehicular traffic, the burning of fossil fuels, wood, coal and activities originated from the civil construction are some of the sources that generate particles. The pollutants particles present in urban area are placed in all of the trees' crowns surface. When washed, part of these particles ends up being incorporated to soil that surrounds the plants, allowing the plants to absorb them.

In huge urban centres, it is proven that the biggest issue is the pollution caused by solid particles, which induces the Earth's cooling by reducing the direct incidence of sunlight. The plants efficiency in slaughtering the pollution effects is associated with the composition, age, position and trees arrangement of the trees used in the urban forestry (Reissmann and Biondi 1994). 
Therefore, in general, the urban trees showed high Fe levels, but, in case of deficiency, in the landscape aspects are affected by the symptoms beginning in the younger leaves with internerval chlorosis, that is, the dark green veins contrasting with the bright green or yellowish surface of the leaves (Mengel and Kirkby 1987, Lilly 2015).

With reference to Mn, authors such as Silveira et al. (2005) indicate that levels lower than 190.00 $\mathrm{mg} \mathrm{kg}{ }^{-1}$ can be inferred as under the crucial concentration that is needed to perform its functions as enzyme activator, participant in the electronic transportation in photosynthesis and producer of chlorophyll and chloroplast.

The contents of Mn demonstrated levels below the satisfactory range in 21 out of 23 species in this study, for C. xanthocarpa $\left(83.85 \mathrm{mg} \mathrm{kg}^{-1}\right), E$. japonica (49.91 mg kg-1), E. involucrata $(25.85$ $\left.\mathrm{mg} \mathrm{kg}{ }^{-1}\right)$, E. uniflora $\left(32.41 \mathrm{mg} \mathrm{kg}^{-1}\right)$, G. robusta (144.15 mg kg-1 $)$ H. heptaphyllus (43.65 mg kg$\left.{ }^{-1}\right)$, I. marginata $\left(85.70 \mathrm{mg} \mathrm{kg}^{-1}\right)$, J. mimosifolia (24.99 $\left.\mathrm{mg} \mathrm{kg}{ }^{-1}\right)$, L. indica $\left(163.43 \mathrm{mg} \mathrm{kg}^{-1}\right)$, M. azedarach (30.04 mg kg-1), M. nigra (88.48 $\left.\mathrm{mg} \mathrm{kg}^{-1}\right), P$. dubium (137.59 $\left.\mathrm{mg} \mathrm{kg}^{-1}\right)$, P. pluviosa $(65.63 \mathrm{mg}$ $\left.\mathrm{kg}^{-1}\right)$, P. cattleyanum $\left(100.16 \mathrm{mg} \mathrm{kg}^{-1}\right), P$. guajava $\left(72.37 \mathrm{mg} \mathrm{kg}^{-1}\right), S$. babylonica $\left(115.54 \mathrm{mg} \mathrm{kg}^{-1}\right)$, S. terebinthifolius (51.39 $\mathrm{mg} \mathrm{kg}^{-1}$ ), S. macranthera (42.77 $\mathrm{mg} \mathrm{kg}^{-1}$ ), S. multijuga (84.49 $\mathrm{mg} \mathrm{kg}^{-1}$ ), $S$. cumini $\left(79.14 \mathrm{mg} \mathrm{kg}^{-1}\right)$ and T. tipu $\left(47.87 \mathrm{mg} \mathrm{kg}^{-}\right.$ $\left.{ }^{1}\right)$, significantly differing only for the species $C$. illinoensis and H. chrysotrichus, which showed acceptable levels.

In the paper of Caldeira et al. (2006), the levels of Mn in A. edulis (160.67 mg kg $\mathrm{mg}^{-1}$, C. xanthocarpa $\left(113.00 \mathrm{mg} \mathrm{kg}^{-1}\right)$, Myrsine ferruginea $(151.00 \mathrm{mg}$ $\left.\mathrm{kg}^{-1}\right)$, Sebastiania brasiliensis $\left(88.00 \mathrm{mg} \mathrm{kg}^{-1}\right)$ and Sebastiania commersoniana (58.00 $\mathrm{mg} \mathrm{kg}^{-1}$ ), were lower than the appropriate level of this nutrient. Reissmann and Carneiro (2004) verified that a higher base saturation decreased the concentration of $\mathrm{Mn}$ in the leaves, in study conducted with the species Ilex paraguariensis. This information can help to explain the low values obtained for most of the species in this study, since Brun et al. (2012) inform that the local soil shows an elevated base saturation.

According to relate by Lilly (2015), the landscape effect of the Mn deficiency can be related to decrease in root growth, which may make the tree more prone to loss of stability and fall, as well as a change in the color of young leaves through internerval chlorosis and necrotic spots, causing the tree to lose its natural landscaping appeal.

Concerning $\mathrm{Zn}$, research papers from Larcher (2000) and Dechen and Nachtigall (2006), argue that concentrations lower than $20 \mathrm{mg} \mathrm{kg}^{-1}$ are under the tolerable amount for the proper growth of tree species. The $\mathrm{Zn}$ levels appeared to be under the adequate rate for E. involucrata $\left(16.82 \mathrm{mg} \mathrm{kg}^{-1}\right), G$. robusta (12.77 $\left.\mathrm{mg} \mathrm{kg}^{-1}\right)$, H. heptaphyllus $(18.03 \mathrm{mg}$ $\left.\mathrm{kg}^{-1}\right)$, J. mimosaefolia (18.99 $\left.\mathrm{mg} \mathrm{kg}^{-1}\right), P$. dubium (19.35 mg kg-1), P. pluviosa (13.23 $\mathrm{mg} \mathrm{kg}^{-1}$ ), $P$. cattleyanum (15.78 $\left.\mathrm{mg} \mathrm{kg}^{-1}\right)$, S. terebinthifolius (17.99 $\left.\mathrm{mg} \mathrm{kg}^{-1}\right)$, S. macranthera (15.79 $\left.\mathrm{mg} \mathrm{kg}^{-1}\right)$, S. multijuga (16.46 $\mathrm{mg} \mathrm{kg}^{-1}$ ) and $S$. cumini $(9.51$ $\mathrm{mg} \mathrm{kg}{ }^{-1}$ ).

Caldeira et al. (2006), analyzing the dynamics of nutrients in Ombrofila Mix Mountain Forest in Parana, acquired, for $\mathrm{Zn}$, limits lower than $20 \mathrm{mg}$ $\mathrm{kg}^{-1}$ for Araucaria angustifolia (16.79 $\mathrm{mg} \mathrm{kg}^{-1}$ ), Colyptranthes concinna (11.76 $\mathrm{mg} \mathrm{kg}^{-1}$ ), Jacaranda puberula (15.70 $\mathrm{mg} \mathrm{kg}^{-1}$ ) Myrcia sp. (8.90 $\left.\mathrm{mg} \mathrm{kg}^{-1}\right)$, Myrsine ferrugine (17.23 mg kg-1), M. umbellata $\left(10.11 \mathrm{mg} \mathrm{kg}^{-1}\right)$, Nectandra megapotonica $(16.30$ $\left.\mathrm{mg} \mathrm{kg}{ }^{-1}\right)$, O. porosa (13.07 $\left.\mathrm{mg} \mathrm{kg}^{-1}\right)$, Prunus brasiliensis (17.96 $\mathrm{mg} \mathrm{kg}^{-1}$ ), S. terebinthifolius (19.22 $\mathrm{mg} \mathrm{kg}^{-1}$ ), S. commersoniana (10.00 mg kg$\left.{ }^{1}\right)$, Sloanea lasiocoma $\left(8.5 \mathrm{mg} \mathrm{kg}^{-1}\right)$ and Styrax leprosus (12.78 $\mathrm{mg} \mathrm{kg}^{-1}$ ). Brun et al. (2012), assessing native tree species planted in urban forestry, got foliar levels for $\mathrm{Zn}$ between 12.92 and $26.74 \mathrm{mg} \mathrm{kg}^{-1}$. Silveira et al. (2005), investigating the foliar concentration levels of distinctive species 
of Eucalyptus sp., concluded that suitable rates for $\mathrm{Zn}$ fluctuated from 12 to $50 \mathrm{mg} \mathrm{kg}^{-1}$.

In most of the tree species employed in the urban forestry of Camobi neighborhood, in Santa Maria - RS, the level of $\mathrm{Zn}$ exposed proper levels. A positive factor regarding the assimilation of this nutrient is that it is plenty located in acid soils (Dechen and Nachtigall 2006), as most of the soils in tropical and subtropical conditions.

In landscape aspects, the $\mathrm{Zn}$ deficiency is different according to the species (Epstein and Bloom 2005) but, in most cases, occurs the formation of rosettes and the leaves become chlorotic. In some species, however, they may become dark green or blue-green, twisted and necrotic. Flowering and fruiting are greatly reduced under conditions of severe $\mathrm{Zn}$ deficiency, and the whole plant may become rickety and misshapen.

The precaution and control limits, upper and lower, of micronutrients and tree species (Table II), in consonance with Lúcio et al. (2010), supply greater conditions to users of laboratorial analyses in evaluating results and make more reliable and consistent decisions with respect to the gathered values.

It is through these nutritional limits that it is possible to question whether the observed results, classified as very high or very low, regarding the precaution and mainly, the control limit, are from contaminated samples or are results of determination errors, measurement or analysis, due to possible calibrations outside the equipment's specifications or negligence of the responsible for the analysis, expressing, in this way, potential lack of control in the analysis process (Caldeira et al. 2006, Lúcio et al. 2010).

Conforming to Feigenbaum (1994), the precaution and control limits settled are utilized to determine the control level of the process, analyze the activity performance, establish the tolerances and the reanalysis costs and define guidelines to the management of the quality control process.
With base on this study (Table II), the nutritional limits showed that the micronutrients in the arboreal species planted in urban areas of South of Brazil can be classified and remedied in situations of deficiency (VL) or toxicity $(\mathrm{VH})$, for best landscaping of urban trees.

\section{CONCLUSIONS}

The concentration of $\mathrm{B}$ resulted a value under the range considered adequate only for G. robusta and showed a higher level than the recommended for $H$. chrysotrichus and M. nigra.

$\mathrm{Cu}$ levels were lower than the satisfactory range for G. robusta and S. cumini.

Fe content performed a high level for $E$. japonica, H. chrysotrichus and S. babylonica.

The foliar levels of Mn demonstrated that this nutrient was under the proper amount for C. xanthocarpa, E. japonica, E. involucrata, E. uniflora, G. robusta, H. heptaphyllus, I. marginata, J. mimosifolia, L. indica, M. azedarach, M. nigra, P. dubium, P. pluviosa, P. cattleyanum, P. guajava, S. babylonica, S. terebinthifolius, S. macranthera, S. multijuga, S. cumini and T. tipu.

The element $\mathrm{Zn}$ performed low levels for E. involucrata, G. robusta, H. heptaphyllus, J. mimosaefolia, P. dubium, P. pluviosa, $P$. cattleyanum, S. terebinthifolius, $S$. macranthera, $S$. multijuga and S. cumini.

$\mathrm{Mn}$ and $\mathrm{Zn}$ were the most crucial elements with reference to the silvicultural performance of the species, and it is necessary to expand the studies and the concern about the fertilization control and management of these trees.

The estimated nutritional limits allow a greater control in the results classification for each tree species utilized in urban forestry in the South Region of Brazil. 


\section{REFERENCES}

ALVARES CA, STAPE JL, SENTELHAS PC, GONÇALVES JLM AND SPAROVEK G. 2013. Koppen's climate classification map for Brazil. Meteorol Z 22(6): 711-728.

BRUN EJ, ROSA SF, ROPPA C, SCHUMACHER MV AND BRUN FGK. 2012. Avaliação nutricional de espécies nativas utilizadas na arborização do campus da Universidade Federal de Santa Maria - RS. REVSBAU, Piracicaba, SP 7(1): 89-111.

CALDEIRA MVW, WATZLAVICK LF, SOARES RV AND VALÉRIO AF. 2006. Teores de Micronutrientes em espécies arbóreas da Floresta Ombrófila Mista Montana General Carneiro/Pr. Ambiência 2(1): 29-50.

COELHO F AND VERLENGIA F. 1973. Fertilidade do solo. $2^{\mathrm{a}}$ ed., Campinas: Instituto Campineiro de Ensino Agrícola, 384 p.

DECHEN AR AND NACHTIGALL GR. 2006. Micronutrientes. In: Fernandes MS (Ed), Nutrição mineral de plantas. Viçosa: Soc Bras Ciência do Solo, p. 327-354.

EMBRAPA. 2006. Sistema Brasileiro de Classificação de Solos. $2^{\mathrm{a}}$ ed., Embrapa, Rio de Janeiro, 306 p.

EPSTEIN E AND BLOOM AJ. 2005. Mineral nutrition of plants: principles and perspectives. $2^{\text {nd }}$ ed., Sunderland: Sinauer Associates.

FEIGENBAUM AV. 1994. Controle da qualidade total: métodos estatísticos aplicados à qualidade. São Paulo: Makson Books, 379 p.

FIRKOWSKI C. 1990. Poluição atmosférica e arborização urbana. In: Encontro Nacional sobre arborização urbana. Curitiba: Fundação de Pesquisas Florestais do Paraná, p. 14-26.

KERN DI AND SCHMITZ JAK. 2013. Arborização de vinte quarteirões amostrados na região central de Santa Cruz do Sul - RS. REVSBAU, Curitiba 8(3): 79-95.

LARCHER W. 2000. Ecofisiologia Vegetal. São Carlos: Rima, $531 \mathrm{p}$.

LILLY SJ. 2015. Guia de estudo para certificação do arborista. ISA, $377 \mathrm{p}$.

LÚCIO AD, STORCK L AND BANZATTO DA. 1999. Classificação dos experimentos de competição de cultivares quanto à sua precisão. Pesq Agrop Gaúcha 5(1): 99-103.

LÚCIO ADC, ROSSATO RAR, SCHUMACHER MV, FORTES FO, STORCK L AND WITSCHORECK R.
2010. Limites de precaução e de controle em análises nutricionais de espécies florestais. Rev Árvore, Viçosa, MG 34(3): 529-537.

MALAVOLTA E, VITTI GC AND OLIVEIRA SA. 1997. Avaliação do estado nutricional das plantas: princípios e aplicações. $2^{\text {a }}$ ed., Piracicaba, POTAFOS, 319 p.

MARSCHNER H. 1995. Mineral nutrition of higher plants. $2^{\text {nd }}$ ed., London: Academic Press.

MENGEL K AND KIRKBY EA. 1987. Principles of plants nutrition. Bern: International Potash Institute.

RAVEN PH, EVERT RF AND EICHHORN SE. 1992. Biologia vegetal. Guanabara Koogan, 905 p.

REISSMANN CB AND BIONDI D. 1994. O teor de ferro em dedaleiro (Lafoensia pacari St. Hill) como elemento indicador da poluição urbana por particulados. Floresta 23(12): 55-62.

REISSMANN CB AND CARNEIRO C. 2004. Crescimento e composição química de Erva-mate (Ilex paraguariensis ST. HIL.), transcorridos oito anos de calagem. Floresta, 34(3): 381-386.

SILVA FAS. 2014. ASSISTAT - Assistência Estatística - versão 7,7 beta (pt), Programa computacional, Universidade Federal de Campina Grande Campus de Campina GrandePB - DEAG/ CTRN.

SILVA FC. 1999. Manual de Análises Químicas de Solos, Plantas e Fertilizantes, Embrapa Solos, $1^{\text {a }}$ edição, Brasília: Editora Embrapa, 370 p.

SILVEIRA RLVA, HIGASHI EN, GONÇALVES NA AND MOREIRA A. 2005. Avaliação do estado nutricional do Eucalyptus: Diagnose visual, foliar e suas interpretações. In: Gonçalves JLM e Benedetti V. Nutrição e fertilização florestal. Piracicaba: IPEF, p. 79-104.

SORREANO MCM, RODRIGUES RR AND BOARETTO AE. 2012. Guia de nutrição para espécies florestais nativas. São Paulo: Oficina de Textos, 254 p.

SZYMCZAK DA, BRUN FGK, BRUN EJ, NAVROSKI MC AND LONDERO EK. 2012. Arborização de vias públicas do Bairro Camobi, Santa Maria, RS. Rev Elet em Gestão, Educação e Tecnologia Ambiental, Santa Maria 8(8): 1611-1625.

TEDESCO MJ, GIANELLO C, BISSANI CA, BOHNEN H AND VOLKWEISS SJ. 1995. Análise de solo, plantas e outros materiais, Porto Alegre: Departamento de Solos, UFRGS, (Boletim Técnico), 118 p. 